\title{
Dopaminergic Modulation of Orofacial Mechanical Hypersensitivity Induced by Infraorbital Nerve Injury
}

\author{
Hiroharu Maegawa *(D, Nayuka Usami, Chiho Kudo, Hiroshi Hanamoto and Hitoshi Niwa \\ Department of Dental Anesthesiology, Osaka University Graduate School of Dentistry, Suita, Osaka 565-0871, \\ Japan; n.adachi@dent.osaka-u.ac.jp (N.U.); ckudo@dent.osaka-u.ac.jp (C.K.); \\ hanamoto@dent.osaka-u.ac.jp (H.H.); niwa@dent.osaka-u.ac.jp (H.N.) \\ * Correspondence: maegawa@dent.osaka-u.ac.jp; Tel.: +81-6-6879-2972; Fax: +81-6-6879-2975
}

Received: 27 January 2020; Accepted: 10 March 2020; Published: 12 March 2020

check for updates

\begin{abstract}
While the descending dopaminergic control system is not fully understood, it is reported that the hypothalamic A11 nucleus is its principle source. To better understand the impact of this system, particularly the A11 nucleus, on neuropathic pain, we created a chronic constriction injury model of the infraorbital nerve (ION-CCI) in rats. ION-CCI rats received intraperitoneal administrations of quinpirole (a dopamine $\mathrm{D} 2$ receptor agonist). ION-CCI rats received microinjections of quinpirole, muscimol [a gamma-aminobutyric acid type $\mathrm{A}\left(\mathrm{GABA}_{\mathrm{A}}\right)$ receptor agonist], or neurotoxin 6-hydroxydopamine (6-OHDA) into the A11 nucleus. A von Frey filament was used as a mechanical stimulus on the maxillary whisker pad skin; behavioral and immunohistochemical responses to the stimulation were assessed. After intraperitoneal administration of quinpirole and microinjection of quinpirole or muscimol, ION-CCI rats showed an increase in head-withdrawal thresholds and a decrease in the number of phosphorylated extracellular signal-regulated kinase (pERK) immunoreactive (pERK-IR) cells in the superficial layers of the trigeminal spinal subnucleus caudalis $(\mathrm{Vc})$. Following 6-OHDA microinjection, ION-CCI rats showed a decrease in head-withdrawal thresholds and an increase in the number of pERK-IR cells in the Vc. Our findings suggest the descending dopaminergic control system is involved in the modulation of trigeminal neuropathic pain.
\end{abstract}

Keywords: chronic constriction injury; infraorbital nerve; A11 nucleus; dopamine receptor; quinpirole; muscimol; 6-hydroxydopamine; phosphorylated extracellular signal-regulated kinase (pERK); trigeminal spinal subnucleus caudalis

\section{Introduction}

The modulation of nociceptive inputs from descending noradrenergic and serotonergic control systems is well documented [1-4]. It is likely that the dopaminergic descending control system is also involved in the processing of nociceptive stimuli $[5,6]$. Several studies showed that the dopaminergic system was related to antinociception [7-9]. However, the descending dopaminergic control system is less well defined [6].

Dopamine receptors are classified into two families: D1-like (D1 and D5) and D2-like (D2, D3, and D4) receptors. The D2-like receptors mediate antinociception $[6,10,11]$ and are the most prominent dopamine receptor subtype in the spinal dorsal horn (SDH) and medullary dorsal horn (MDH) [12-14].

The hypothalamic A11 nucleus is the principle source of descending dopaminergic pathways [6]. The A11 nucleus has projections to the MDH [15], and local electrical or pharmacological stimulation of the A11 nucleus inhibits responses of SDH/MDH neurons to noxious stimulation, through the activation of segmental D2 receptors $[10,16]$. On the other hand, electrical lesions of the A11 nucleus 
facilitate responses of neurons in the trigeminocervical complex to both noxious and innocuous mechanical stimuli [16]. Administration of quinpirole (a dopamine D2 receptor agonist) to the A11 nucleus attenuates mechanical hypersensitivity in rats with spinal nerve ligation [17]. Stimulation of the A11 nucleus attenuates trigeminal neuropathic pain in mice [18].

However, the function of the A11 nucleus is not fully revealed. The aim of the present study was to assess: (1) the effects of administration of a dopamine D2 receptor agonist on trigeminal neuropathic pain and (2) the effects of the A11 nucleus on trigeminal neuropathic pain to better understand the role of the descending dopaminergic control system. In the present study, we created a chronic constriction injury model of the infraorbital nerve (ION-CCI) in rats as the trigeminal neuropathic pain model. Mechanical stimulation by von Frey filament to the maxillary whisker pad skin was used to generate a behavioral response (the head-withdrawal threshold) and an immunohistochemical response [phosphorylated extracellular signal-regulated kinase (pERK) expression in the superficial layers of the trigeminal spinal subnucleus caudalis $(\mathrm{Vc})]$ following intraperitoneal (i.p.) administration of quinpirole in ION-CCI rats. We used the same methods to evaluate the effects of microinjections of quinpirole, muscimol [a gamma-aminobutyric acid type $\mathrm{A}\left(\mathrm{GABA}_{\mathrm{A}}\right)$ receptor agonist], or neurotoxin 6-hydroxydopamine (6-OHDA) into the A11 nucleus in ION-CCI rats to assess the role of the A11 nucleus on trigeminal neuropathic pain.

\section{Results}

\subsection{The Change of Head-Withdrawal Threshold following ION-CCI}

Rats received mechanical stimulation by von Frey filaments to the maxillary whisker pad skin on the ipsilateral side to nerve ligation to measure head-withdrawal threshold. The results of the test of the head-withdrawal threshold showed a significant interaction $[\mathrm{F}(4,40)=8.20, p<0.001]$. Rats with ION-CCI $(n=6)$ showed mechanical hypersensitivity from 3 to 21 days after nerve ligation; they showed a significant decrease in head-withdrawal thresholds as compared with sham-operated rats on $3,7,14$, and 21 days after ION-CCI ( 7 days after: $p<0.001 ; 3$ days after: $p=0.002 ; 14$, and 21 days after: $p=0.001$, Figure 1, File S1). Rats with ION-CCI showed a significant decrease in head-withdrawal thresholds to mechanical stimulation 3 days after ION-CCI as compared with before ION-CCI; this decrease lasted until 21 days after nerve ligation ( 3 and 7 days after: $p<0.001,14$ and 21 days after: $p=0.001$, Figure 1). Sham operated rats $(n=6)$ showed no significant change in head-withdrawal threshold (Figure 1).

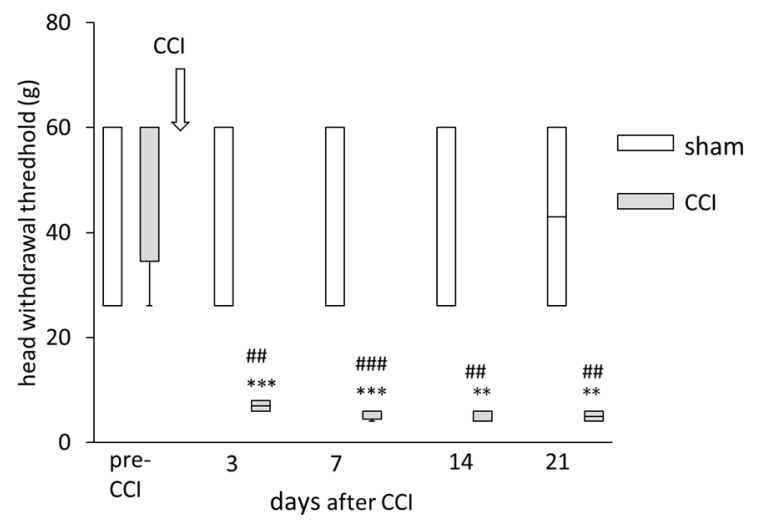

Figure 1. The time course of changes in head-withdrawal threshold of chronic constriction injury of the infraorbital nerve (ION-CCI) in rats. Data are shown as box and whisker plots. The bottom and top of the box indicate the first and third quartiles, respectively. The end of the whiskers represents the minimum and maximum values of all data. Rats with ION-CCI showed mechanical hypersensitivity from 3 to 21 days after nerve ligation; they showed a significant decrease in head-withdrawal thresholds 
as compared with sham-operated rats on 3, 7, 14, and 21 days after ION-CCI. Rats with ION-CCI showed a significant decrease in head-withdrawal thresholds to mechanical stimulation 3 days after ION-CCI as compared with before ION-CCI; this decrease lasted until 21 days after nerve ligation. ${ }^{* * *} p<0.001$ compared with head-withdrawal threshold before ION-CCI. ${ }^{* *} p<0.01$ compared with head-withdrawal threshold before ION-CCI. ${ }^{\# \#} p<0.001$ compared with head-withdrawal threshold of sham-operated rats on the same time. ${ }^{\# \#} p<0.01$ compared with head-withdrawal threshold of sham-operated rats on the same time.

2.2. The Effect of Systemic Administration of Quinpirole on Mechanical Hypersensitivity and $p E R K$ Expression in the Superficial Layers of the Vc

The results of the test of the head-withdrawal threshold showed a significant interaction $[\mathrm{F}(5,60)=13.19, p<0.001]$. Fourteen days after ION-CCI, ION-CCI rats that received i.p. administration of quinpirole $(n=7)$ showed a significant increase in head-withdrawal thresholds as compared with those before drug administration. The increase was found 20, 60, 120, 180, and $240 \mathrm{~min}$ after the administration of quinpirole $(20,60,120$, and $180 \mathrm{~min}$ after: $p<0.001 ; 240 \mathrm{~min}$ after: $p=0.001$; Figure 2A; Supplementary File S1). On the other hand, ION-CCI rats that received i.p. administration of saline $(n=7)$ showed no significant change of head-withdrawal threshold as compared with those before saline administration. The head-withdrawal threshold of ION-CCI rats with i.p. administration of quinpirole was larger than that of ION-CCI rats with i.p. administration of saline at 20,60, 120, 180, and $240 \mathrm{~min}$ after administration, respectively $(20,60,120$, and180 min after: $p<0.001 ; 240 \mathrm{~min}$ after: $p=0.001$; Figure 2A). Twenty minutes after quinpirole or saline administration, we also applied mechanical stimulation by von Frey filament $(15 \mathrm{~g}, 1 \mathrm{~Hz}, 5 \mathrm{~min})$ to the maxillary whisker pad skin on the ipsilateral side to nerve ligation in ION-CCI rats ( $n=7$ in each group). Five minutes after the stimulation, rats were perfused, and then, immunohistochemical staining for pERK in the Vc was performed (Figure 2B, Figures S1 and S2). The greatest number of labeled cells that were pERK immunoreactive (pERK-IR) were found in the superficial layers of the $\mathrm{Vc}_{\mathrm{c}} 1.4 \mathrm{~mm}$ caudal to the obex in both groups. The number of pERK-IR cells/section in the superficial layers of the Vc of ION-CCI rats that received quinpirole administrations was smaller than that of ION-CCI rats that received saline administrations ( $16.8 \pm 2.4$ vs. $27.7 \pm 2.7, \mathrm{df}=68, t=7.36, p<0.001$, Figure $2 \mathrm{C}$, Supplementary File S1).

A

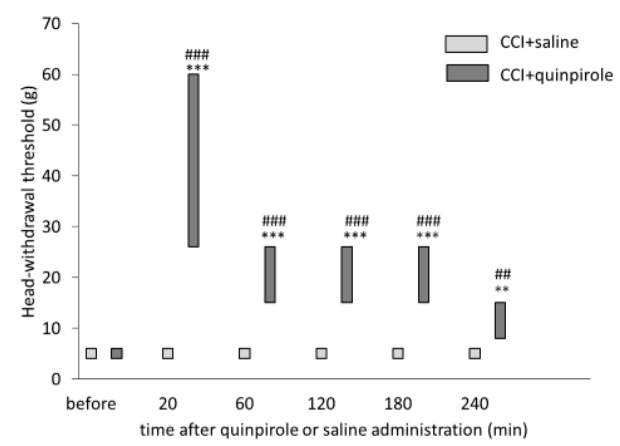

B
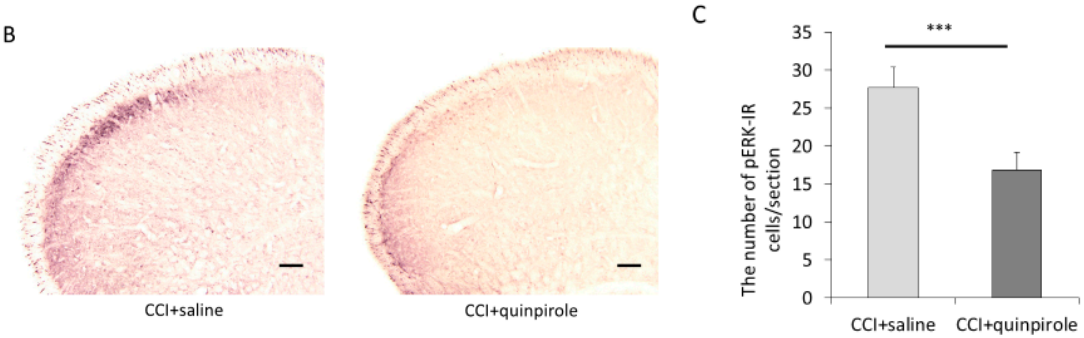

Figure 2. Effects of intraperitoneal (i.p.) administration of quinpirole $(1 \mathrm{mg} / \mathrm{kg})$ on ION-CCI. (A) The change in head-withdrawal threshold after quinpirole administration. Data are shown as box and 
whisker plots. The bottom and top of the box indicate the first and third quartiles, respectively. The end of the whiskers represents the minimum and maximum values of all data. Fourteen days after ION-CCI, ION-CCI rats that received i.p. administration of quinpirole showed a significant increase in head-withdrawal thresholds 20,60, 120, 180, and $240 \mathrm{~min}$ after the administration of quinpirole as compared with thresholds before drug administration. ${ }^{* *} p<0.001$ compared with head-withdrawal threshold before administration. ${ }^{* *} p<0.01$ compared with head-withdrawal threshold before administration. The head-withdrawal threshold of ION-CCI rats with i.p. administration of quinpirole was larger than that of ION-CCI rats with i.p. administration of saline at 20,60, 120, 180, and 240 min after administration. ${ }^{\# \#} p<0.001$ compared with head-withdrawal threshold of ION-CCI rats that received saline administration at the same time. ${ }^{\# \#} p<0.01$ compared with head-withdrawal threshold of ION-CCI rats that received saline administration at the same time. (B) Photomicrograph of phosphorylated extracellular signal-regulated kinase (pERK) in the superficial layers of the trigeminal spinal subnucleus caudalis (Vc) of ION-CCI rats that received saline or quinpirole administrations. Scale bar represents $100 \mu \mathrm{m}$. (C) The number of pERK immunoreactive (pERK-IR) cells/section in the Vc (mean $\pm \mathrm{SEM}$ ). The number of pERK-IR cells/section in the Vc of ION-CCI rats that received quinpirole administrations was significantly larger than that of ION-CCI rats that received saline administrations. $* * * p<0.001$.

\subsection{The Effect of Microinjections of Quinpirole and Muscimol into the A11 Nucleus on Mechanical} Hypersensitivity and $p E R K$ Expression in the Superficial Layers of the $V_{c}$

Seven days after ION-CCI, a metal cannula was implanted into the skull of ION-CCI rats for a microinjection of drugs. ION-CCI rats that had a metal cannula implanted into the skull exhibited no significant change in head-withdrawal threshold between before (7 days after ION-CCI) and after the implantation procedure (13 days after ION-CCI) (Supplementary File S1).

Fourteen days after ION-CCI (7 days after the implantation), we administered quinpirole or muscimol or saline through the cannula (Figure 3A, Figures S3 and S4). Injection sites were distributed $-3.3 \mathrm{~mm}$ to $-3.9 \mathrm{~mm}$ from bregma (Figure $3 \mathrm{~B}$ ). The number of misplacements of cannula were as follows: ION-CCI rats with saline microinjection: 2, ION-CCI rats with quinpirole microinjection: 2, and ION-CCI rats with muscimol microinjection: 3 . The results of the test of the head-withdrawal threshold showed a significant interaction $[\mathrm{F}(6,54)=14.38, p<0.001]$. The head-withdrawal threshold in ION-CCI rats that received microinjections of quinpirole $(n=7)$ was larger than that in ION-CCI rats that received microinjections of saline $(n=7) 20$ and $40 \mathrm{~min}$ after the microinjection (20 min: $p<0.001,40$ min: $p=0.004$, Figure 3C). The head-withdrawal threshold in ION-CCI rats that received microinjections of muscimol $(n=7)$ was larger than that in ION-CCI rats that received microinjections of saline 20 and $40 \mathrm{~min}$ after the microinjection $(p<0.001$, Figure 3C). ION-CCI rats that received microinjections of quinpirole showed a significant increase in head-withdrawal thresholds 20 and 40 min after microinjections as compared with thresholds before microinjections $(p<0.001$, Figure 3C, Supplementary File S1). ION-CCI rats that received microinjections of muscimol also showed a significant increase in head-withdrawal thresholds 20 and $40 \mathrm{~min}$ after microinjections as compared with thresholds before microinjections $(p<0.001$, Figure 3C). Twenty minutes after quinpirole, muscimol, or saline microinjection, we also applied mechanical stimuli by von Frey filament (15 g, $1 \mathrm{~Hz}, 5 \mathrm{~min}$ ) to ION-CCI rats as described above ( $\mathrm{n}=7$ in each group). As with the i.p. administrations, after mechanical stimulation and immunohistochemistry, the greatest number of pERK-IR cells were found in the superficial layers of the Vc $1.4 \mathrm{~mm}$ caudal to the obex in all groups (Figure 3D, Figures S5, S6 and S7). As a result of a test of the number of pERK-IR cells in the $\mathrm{V}_{\mathrm{c}}$, the main effect was found to be significant $[F(2,102)=64.17, p=0.001]$. The mean number of pERK-IR cells/section in the Vc of ION-CCI rats that received microinjections of quinpirole was smaller than that of ION-CCI rats that received microinjections of saline $(15.9 \pm 0.4$ vs. $27.0 \pm 0.7, p<0.001$, Figure 3E, Supplementary File S1). The mean number of pERK-IR cells/section in the Vc of ION-CCI rats that received microinjections of muscimol was also smaller than that of ION-CCI rats that received microinjections of saline $(12.9 \pm 1.7$ vs. $20.7 \pm 0.7, p<0.001$, Figure $3 \mathrm{E})$. 
A
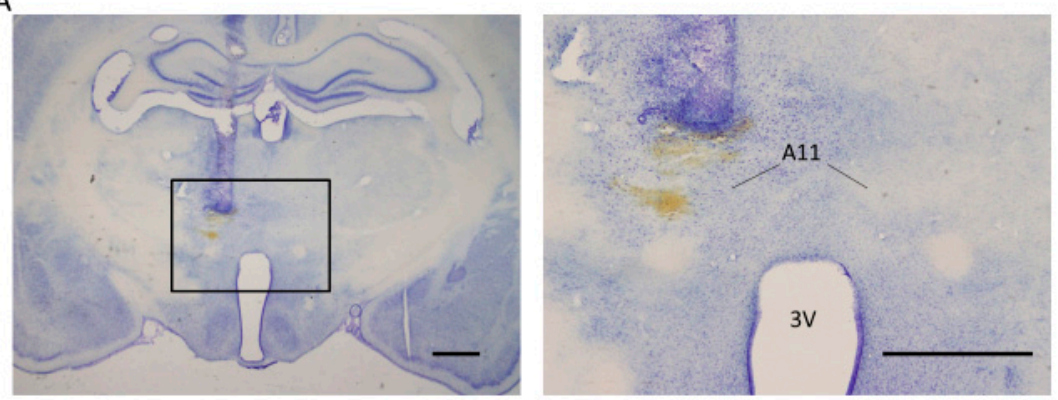

B

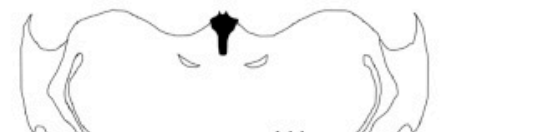

C
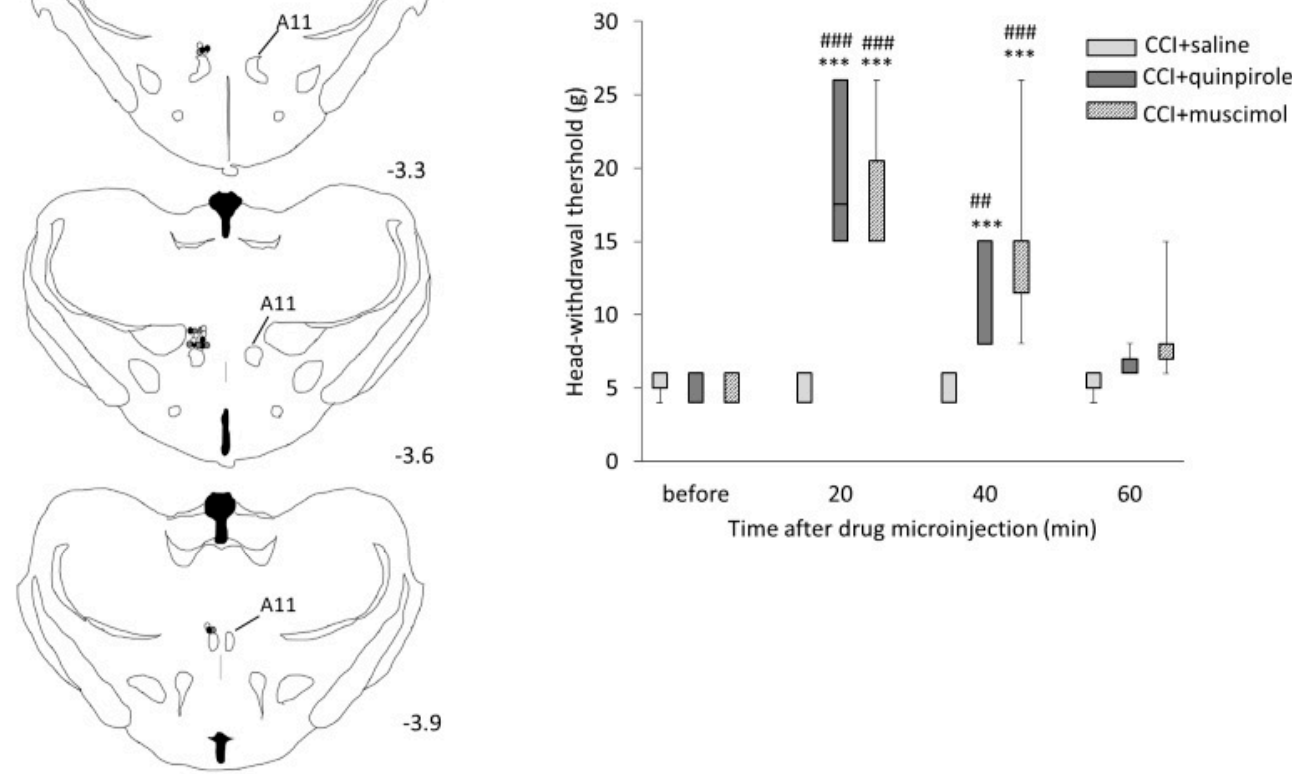

D

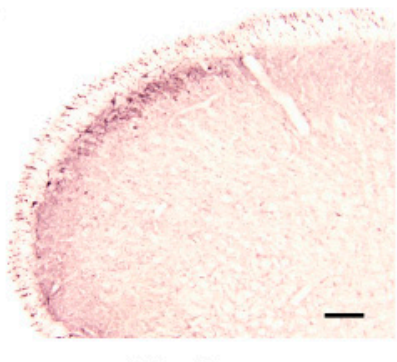

CCl+saline

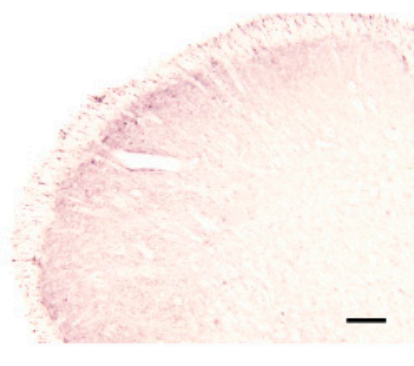

$\mathrm{CCl}+$ quinpirole

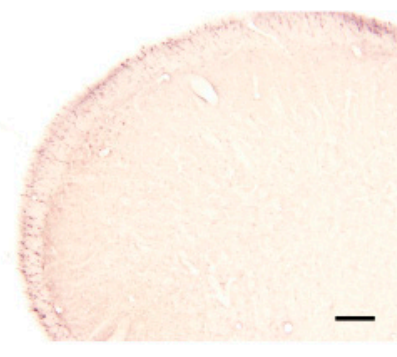

$\mathrm{CCl}+$ muscimol

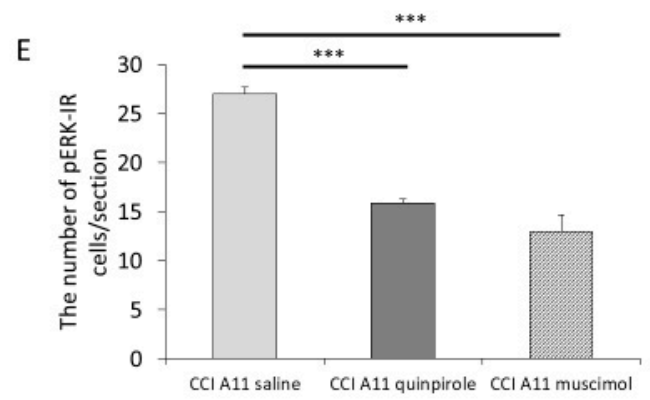

Figure 3. Effects of microinjection of quinpirole $(20 \mu \mathrm{g}$ in $0.25 \mu \mathrm{L})$ and muscimol $(0.07 \mu \mathrm{g}$ in $0.25 \mu \mathrm{L})$ into the A11 nucleus of ION-CCI. (A) Photomicrograph of the injection site within the A11 nucleus at low and high magnification. Scale bar represents $1 \mathrm{~mm}$. 3V: third ventricle. (B) Injection site into the 
A11 nucleus (white circle: saline, gray circle: quinpirole, black circle: muscimol). Digit represents a distance from bregma (mm). (C) The change in head-withdrawal threshold after microinjections of quinpirole or muscimol. Data are shown as box and whisker plots. The bottom and top of the box indicate the first and third quartiles, respectively. The end of the whiskers represents the minimum and maximum values of all data. ION-CCI rats that received microinjections of quinpirole showed a significant increase in head-withdrawal threshold 20 and $40 \mathrm{~min}$ after the microinjection, as compared with thresholds before the microinjection. ION-CCI rats that received microinjections of muscimol showed a significant increase in head-withdrawal threshold 20 and $40 \mathrm{~min}$ after the microinjection as compared with thresholds before microinjections. The head-withdrawal threshold in ION-CCI rats that received microinjections of quinpirole was larger than that in ION-CCI rats that received microinjections of saline 20 and $40 \mathrm{~min}$ after the microinjection. The head-withdrawal threshold in ION-CCI rats that received microinjections of muscimol was larger than thresholds in ION-CCI rats that received microinjections of saline 20 and 40 min after the microinjection. ${ }^{* * *} p<0.001$ compared with head-withdrawal threshold before microinjections. ${ }^{* *} p<0.01$ compared with head-withdrawal threshold before microinjections. ${ }^{\# \#} p<0.001$ compared with head-withdrawal threshold of ION-CCI rats that received saline microinjections at the same time. ${ }^{\# \#} p<0.01$ compared with head-withdrawal threshold of ION-CCI rats that received saline microinjections at the same time. (D) Photomicrographs of pERK-IR cells in the Vc of ION-CCI rats that received microinjections of saline, quinpirole, or muscimol into the A11 nucleus. Scale bar represents $100 \mu \mathrm{m}$. (E) The number of pERK-IR cells/section in the $\mathrm{V}_{\mathrm{c}}($ mean $\pm \mathrm{SEM})$. The number of pERK-IR cells/section in the $\mathrm{V}_{\mathrm{c}}$ of ION-CCI rats that received microinjections of quinpirole was smaller than that of ION-CCI rats that received microinjections of saline. The number of pERK-IR cells/section in the Vc of ION-CCI rats that received microinjections of muscimol was smaller than that of ION-CCI rats that received microinjections of saline. ${ }^{* * *} p<0.001$.

2.4. The Effect of Microinjections of 6-OHDA into the A11 Nucleus on Mechanical Hypersensitivity and pERK Expression in the Superficial Layers of the Vc

Seven days after ION-CCI, ION-CCI rats received microinjections of 6-OHDA or saline into the A11 nucleus. Fourteen days after 6-OHDA microinjection (21 days after ION-CCI), immunohistochemical staining for tyrosine hydroxylase $(\mathrm{TH})$ was performed in all the rats that were microinjected with 6-OHDA or saline into the A11 nucleus ( $n=14$ in each group, Figure 4A). Injection sites were distributed $-3.3 \mathrm{~mm}$ to $-3.9 \mathrm{~mm}$ from bregma (Figure $4 \mathrm{~B}$ ). The number of misplacements of cannula were as follows: ION-CCI rats with saline microinjection: 3 and ION-CCI rats with 6-OHDA microinjection: 4. ION-CCI rats that received 6-OHDA or saline microinjections showed TH immunoreactive (TH-IR) cells throughout the entire rostrocaudal extent of the A11 nucleus. The number of TH-IR cells was large in the rostral part of the A11 nucleus and gradually decreased toward the caudal end. As a result of a test of the number of TH-IR cells in the A11 nucleus, a significant interaction was found $[F(1,52)=18.13$, $p<0.001]$. In ION-CCI rats with 6-OHDA microinjections, the significant decrease of TH-IR cells in the A11 nucleus was found in the ipsilateral side to 6-OHDA microinjection as compared with the contralateral side (11.9 \pm 1.2 vs. $24.3 \pm 1.0, p<0.001$, Figure 4C, File S1, Figures S8 and S9). ION-CCI rats with 6-OHDA microinjections showed approximately $40 \%$ fewer TH-IR cells in the A11 nucleus on the ipsilateral side to microinjections as compared with the ipsilateral side in ION-CCI rats with saline microinjections $(11.9 \pm 1.2$ vs. $29.2 \pm 1.0, p<0.001$, Figure $4 \mathrm{C})$. The results of the test of the head-withdrawal threshold showed a significant interaction $[\mathrm{F}(1,12)=10.8, p<0.01]$. At 14 days after 6-OHDA microinjection (21 days after ION-CCI), ION-CCI rats that received microinjections of 6-OHDA into the A11 nucleus $(n=7)$ showed a significant decrease in head-withdrawal thresholds as compared with thresholds of 7 days after ION-CCI (before 6-OHDA microinjection) $(p<0.001$, Figure 4D, Supplementary File S1). At 21 days after ION-CCI, the head-withdrawal threshold of ION-CCI rats that received microinjections of 6-OHDA into the A11 nucleus was smaller than that of ION-CCI rats that received microinjections of saline $(n=7, p=0.004$, Figure $4 \mathrm{D})$. We also applied mechanical stimuli by von Frey filament $(15 \mathrm{~g}, 1 \mathrm{~Hz}, 5 \mathrm{~min})$ to ION-CCI rats as described above $(n=7$ 
in each group). The greatest number of pERK-IR cells were found in the superficial layers of the $\mathrm{Vc}$ $1.4 \mathrm{~mm}$ caudal to the obex in both groups (Figure 4E, Figures S10 and S11). The mean number of pERK-IR cells/section in the Vc was larger in ION-CCI rats that received microinjections of 6-OHDA as compared with ION-CCI rats that received microinjections of saline ( $32.4 \pm 6.6$ vs. $26.0 \pm 2.1, \mathrm{df}=68$, $t=3.06, p<0.05$, Figure 4 F, Supplementary File S1).
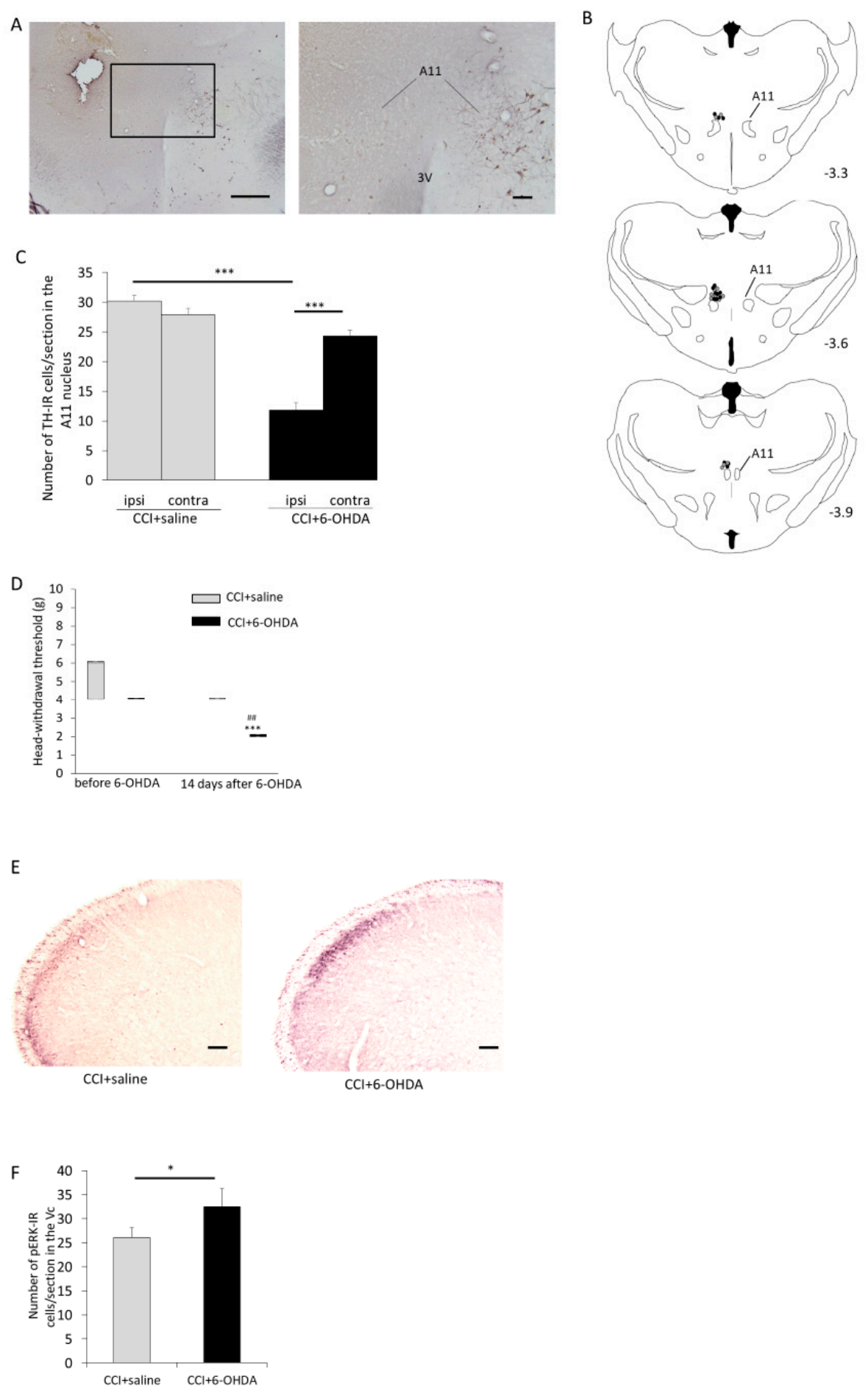

Figure 4. Effects of microinjection of 6-OHDA ( $2 \mathrm{mg} / \mathrm{mL}, 1 \mu \mathrm{L} / \mathrm{min}$ for $1 \mathrm{~min})$ into the A11 nucleus on ION-CCI. (A) Photomicrograph of the A11 nucleus at low and high magnification. The immunoreactivity of tyrosine hydroxylase (TH) decreased on the ipsilateral side to 6-OHDA microinjection. Scale bar represents $500 \mu \mathrm{m}$ (low magnification) and $100 \mu \mathrm{m}$ (high magnification). 3V: third ventricle. (B) Injection 
site into the A11 nucleus (gray circle: saline, black circle: 6-OHDA). Digit represents a distance from bregma (mm). (C) The mean number of TH immunoreactive (TH-IR) cells/section in the A11 nucleus (mean \pm SEM). In ION-CCI rats with 6-OHDA microinjections, a significant decrease of TH-IR cells in the A11 nucleus was found in the ipsilateral side to 6-OHDA microinjection as compared with contralateral side. ION-CCI rats with 6-OHDA microinjections showed a significant decrease in TH-IR cells in the A11 nucleus on the ipsilateral side to 6-OHDA microinjection as compared with the ipsilateral side in ION-CCI rats with saline microinjections. ${ }^{* * *} p<0.001$. (D) The change in head-withdrawal threshold after microinjection of 6-OHDA. Data are shown as box and whisker plots. The bottom and top of the box indicate the first and third quartiles, respectively. The end of the whiskers represents the minimum and maximum values of all data. ION-CCI rats that received 6-OHDA microinjections into the A11 nucleus showed a significant decrease in head-withdrawal thresholds as compared with thresholds 7 days after ION-CCI (before 6-OHDA microinjections). ION-CCI rats that received 6-OHDA microinjections into the A11 nucleus showed a significant decrease in head-withdrawal thresholds as compared with ION-CCI rats that received saline microinjections 14 days after 6-OHDA microinjections (21 days after ION-CCI). ${ }^{* * *} p<0.001$ compared with head-withdrawal threshold before 6-OHDA microinjections. ${ }^{\#} p<0.01$ compared with head-withdrawal threshold in ION-CCI rats with saline microinjections. (E) Photomicrograph of pERK-IR cells in the Vc of ION-CCI rats that received microinjections of saline or 6-OHDA into the A11 nucleus. Scale bar represents $100 \mu \mathrm{m}$. (F) The mean number of $\mathrm{pERK}-I \mathrm{R}$ cells/section in the $\mathrm{Vc}$ (mean $\pm \mathrm{SEM})$. The mean number of $\mathrm{pERK}-\mathrm{IR}$ cells/section in the $\mathrm{Vc}$ was larger in ION-CCI rats that received microinjections of 6-OHDA as compared with ION-CCI rats that received microinjections of saline. ${ }^{*} p<0.05$.

\section{Discussion}

We found that the systemic administration of a dopamine D2 receptor agonist attenuated the mechanical hypersensitivity induced by ION-CCI and decreased the number of pERK-IR cells in the superficial layers of the $\mathrm{Vc}$ in rats with ION-CCI. Following stimulation, pERK expression reaches to a peak within $3 \mathrm{~min}$ [19]. Previously, ION-CCI rats were perfused $5 \mathrm{~min}$ after stimulation, and pERK expression in the Vc was investigated [20]. Therefore, we perfused rats $5 \mathrm{~min}$ after mechanical stimulation to the maxillary whisker pad skin according to the previous report [20]. Our findings indicate that systemic administration of a dopamine D2 receptor agonist suppresses mechanical hypersensitivity induced by ION-CCI. Previously, systemic administration of dopamine D2 or D2/D3 receptor agonists was reported to attenuate pain-related behavior in control [21-25] and hypersensitivity conditions induced by intraplanter injection of carrageenan [26] and sciatic nerve ligation [27]. Previous work has shown that neuropathic pain rat models that received i.p. administration of quinpirole showed an increase in withdrawal threshold to mechanical stimuli [27]. While the applied drug dose was the same in both studies, the methodology was different with the previous study using rats with sciatic nerve ligation, as opposed to our rats that had infraorbital nerve ligation; in addition, different measurement techniques were used. This might explain why the previous work reported an increase in withdrawal threshold that persisted for more than $24 \mathrm{~h}$, but our rats with ION-CCI showed an increase in head-withdrawal threshold for $180 \mathrm{~min}$.

The A11 nucleus is known to have a high density of dopamine D2 receptors [28]. In the present study, we found that microinjections of a dopamine D2 receptor agonist into the A11 nucleus attenuated the mechanical hypersensitivity induced by ION-CCI and decreased the number of pERK-IR cells in the $\mathrm{Vc}_{\mathrm{c}}$ in ION-CCI rats. Our results show that microinjections of a dopamine D2 receptor agonist into the A11 nucleus change the activity of the A11 nucleus and inhibit the activity of the Vc neurons in ION-CCI rats, suggesting that the A11 nucleus is involved in descending modulation of mechanical hypersensitivity induced by ION-CCI through dopamine D2 receptors. Microinjection of a dopamine D2 receptor agonist into the A11 nucleus attenuated mechanical hypersensitivity induced by spinal nerve ligation in a previous study [17]; in our work, we found that the duration of the increase in head-withdrawal threshold to mechanical stimuli was longer in ION-CCI rats that received i.p. 
administration of quinpirole as compared with ION-CCI rats that received microinjections of quinpirole directly into the A11 nucleus. The presence of bilateral projections from the A11 nucleus to MDH is reported [15]. The expression of dopamine D2 receptors is indicated in the $V_{c}[13,18,29]$. Additionally, microinjections of quinpirole into the $\mathrm{MDH}$ is reported to elicit the depression of c-fiber evoked responses [29]; this finding suggests the inhibition of $V_{c}$ neurons by the activation of dopamine D2 receptors in the Vc. Taken together, these findings suggest that dopamine released from dopaminergic neurons in the A11 nucleus modulate the activity of the $\mathrm{V}_{\mathrm{c}}$ neurons through dopamine D2 receptors expressed in the Vc.

Administration of dopamine D2 receptor agonists to regions external to the $V_{c}$ has been shown to suppress neuropathic pain. Striatal administration of dopamine D2 receptor agonist was reported to attenuate withdrawal responses to mechanical stimuli in rats with peroneal and tibial nerve ligations [30]. Intrathecal administration of a dopamine D2 receptor agonist was reported to inhibit allodynic responses to mechanical stimuli in rats with sciatic nerve ligation [27]. Thus, it is likely that when dopamine D2 receptor agonists are administered intraperitoneally, they can act thorough not only the A11 nucleus but also other regions, such as the striatum or intrathecal space. Alleviation of mechanical hypersensitivity following i.p. administration of dopamine D2 receptor agonists may therefore be a mixture of effects that occur through these regions.

Our findings suggest that microinjections of a $\mathrm{GABA}_{\mathrm{A}}$ receptor agonist into the A11 nucleus change the activity of the A11 nucleus and inhibit the activity of $\mathrm{V}_{c}$ neurons in ION-CCI rats. These findings also suggest that the A11 nucleus is involved in descending modulation of trigeminal neuropathic pain. Microinjections of a $\mathrm{GABA}_{\mathrm{A}}$ receptor agonist into the A11 nucleus have been previously shown to attenuate mechanical hypersensitivity induced by spinal nerve ligation [17]; microinjections of a $\mathrm{GABA}_{\mathrm{A}}$ receptor agonist into the $\mathrm{A} 11$ nucleus also reduced rubbing responses to subcutaneous formalin injection into the upper lip of rats [15]. We found that ION-CCI rats that received microinjections of 6-OHDA into the A11 nucleus exhibited a decrease in head-withdrawal thresholds and an increase in the number of pERK-IR cells in the Vc. These findings suggest that the destruction of dopaminergic cells in the A11 nucleus exacerbates mechanical hypersensitivity induced by ION-CCI. Inhibition of the A11 nucleus by muscimol alleviated mechanical hypersensitivity induced by ION-CCI and the destruction of dopaminergic cells by 6-OHDA in the A11 nucleus exacerbated mechanical hypersensitivity. In the A11 nucleus, there are three types of neurons: dopamine-, GABA-, and calcitonin gene-related peptide (CGRP)-containing neurons [31,32]. Taken together, these results suggest that GABA- or CGRP-containing neurons in the A11 nucleus may be involved in the alleviation of mechanical hypersensitivity induced by ION-CCI. The A11 nucleus contains almost 2.5 times more GABA-containing neurons as compared with dopamine-containing neurons, and noxious facial stimulation activates GABA-containing neurons in the A11 nucleus [15], suggesting that GABA-containing neurons in the A11 nucleus might be involved in the regulation of dopaminergic neuron activity. Dopamine D2 agonist and $\mathrm{GABA}_{\mathrm{A}}$ receptor agonist were reported to affect motor function [33-36]. Therefore, the change of head-withdrawal threshold measured in our study might be the result of not only attenuation of mechanical hypersensitivity but also change of motor function by quinpirole and muscimol. This point could be a limitation of the present study.

Microinjections of 6-OHDA into the A11 nucleus were reported to decrease face rubbings induced by formalin injection into the upper lip of normal rats [15] and increase head-withdrawal threshold to mechanical stimuli with von Frey filament in ION-CCI mice [18]. The former showed approximately $40 \%$ decrease in TH-IR cells, and the latter showed approximately $70 \%$ decrease. Dopamine is known to have a higher affinity to D2-like receptors and activates them with lower concentrations of dopamine than D1-like receptors [37]. Therefore, low levels of dopamine could activate D2-like receptors leading to inhibition of pain, and high levels of dopamine would activate D1-like receptors leading to exacerbation of pain [38]. Furthermore, even high levels of dopamine could activate D2-like receptors due to a shift of preference to D2-like receptors [37,39]. Protein of dopamine D2 receptors are increased in the dorsal horn of rats with spinal nerve ligation [40], which suggests that a change in expression 
of dopamine receptors may occur between normal and neuropathic pain model rats. For our study, as well as others [15,18], the number of TH-IR neurons, not dopamine levels, was investigated, so it remains unclear whether the decrease in TH-IR neurons reflects the decrease in dopamine levels.

A previous report indicated that mechanical hypersensitivity induced by ION-CCI lasted 42 days after nerve ligation [41]. Heat hypersensitivity was also reported [27,42], and it lasted 12 days after ION-CCI [42]. A previous report also indicated that intrathecal coadministration of quinpirole and $\mu$-opioid receptor agonist attenuated mechanical and heat hypersensitivity induced by sciatic nerve ligation [43]. Increases of dopamine D2 receptors and their colocalization with $\mu$-opioid receptors were reported in the dorsal horn during pathological process of pain $[44,45]$. A similar reaction may occur in the $\mathrm{Vc}$ of ION-CCI rats.

In summary, we showed that dopamine D2 agonist attenuated mechanical hypersensitivity induced by ION-CCI. We suggested that the A11 nucleus is involved in the modulation of mechanical hypersensitivity induced by infraorbital nerve ligation in rats through a modulation of activities of the Vc neurons. These findings help uncover the characteristics of the descending dopaminergic system and may lead to further alleviation of neuropathic pain.

\section{Material and Methods}

Protocols in the present study were performed in accordance with the ethical guidelines of the International Association for the Study of Pain [46] and were approved by the Osaka University Graduate School of Dentistry Animal Care and Use Committee (29-003, 9 May 2017).

\subsection{Infraorbital Nerve Constriction}

Male Wistar rats (SLC, Hamamatsu, Japan) were housed under a $12 \mathrm{~h}$ dark/light cycle with ad libitum access to food and water. Rats ranged from 170 to $200 \mathrm{~g}$ at the beginning of the experiment and were anesthetized by i.p. administration of saline solution mixed with $2.0 \mathrm{mg} / \mathrm{kg}$ midazolam (Sandoz, Tokyo, Japan), $2.5 \mathrm{mg} / \mathrm{kg}$ butorphanol (Meiji Seika Pharma, Tokyo, Japan), and $0.375 \mathrm{mg} / \mathrm{kg}$ medetomidine (Zenoaq, Fukushima, Japan). Rats were placed in the supine position and a small intra oral incision was made along the gingiva-buccal margin proximal to the first molar, exposing the left ION [42]. The ION was ligated loosely with two ligatures using 4-0 silk to create a chronic constriction injury of the ION. For control animals, a sham operation was performed without nerve ligation.

\subsection{Measurement of Mechanical Head-Withdrawal Threshold}

Rats were trained every day to keep their snout protruding from a plastic cage with a small hole on the front wall during mechanical stimulation to the maxillary whisker pad skin. Rats could escape from applied stimulation freely. Once training has been successfully completed, mechanical head-withdrawal threshold was measured using von Frey filaments. Applied mechanical stimuli with von Frey filaments were $0.008,0.02,0.04,0.07,0.16,0.4,0.6,1,1.4,2,4,6,8,10,15,26,35,45$, and $60 \mathrm{~g}$ (North Coast Medical, Morgan Hill, CA, USA). The maximum force of $60 \mathrm{~g}$ was used to prevent tissue damage [47]. Mechanical stimuli were applied to the maxillary whisker pad skin of rats on the ipsilateral side to ION-CCI. Head-withdrawal threshold was determined by the minimum force that elicited a withdrawal response to greater than three out of five stimuli. We used six rats with ION-CCI to investigate the time course of mechanical hypersensitivity following ION-CCI. Measurements of the head-withdrawal threshold were determined before and 3, 7, 10, 14, and 21 days after nerve ligation. Rats with ION-CCI and a head-withdrawal threshold below $6 \mathrm{~g}$ at 7 days after nerve ligation were categorized as ION-CCI rats. For the subsequent experiments, performed 7 to 21 days after nerve ligation, we used rats that showed a decrease in head-withdrawal threshold (below $6 \mathrm{~g}$ ) at 7 days after ION-CCI. Seven days after ION-CCI, 42 rats were implanted with metal cannulas to the skull for microinjection of drugs, and 28 rats received microinjections of 6-OHDA into the A11 nucleus following the measurement of the head-withdrawal threshold. For rats that received the implantation of a cannula, we measured head-withdrawal threshold 13 days after ION-CCI, just before 
subsequent experiments were performed 14 days after ION-CCI. These rats still showed a decrease in head-withdrawal threshold (below $6 \mathrm{~g}$ ) 13 days after ION-CCI. Measurement of head-withdrawal threshold was performed using a blind protocol, so that the investigator measuring head-withdrawal thresholds was unaware of which experimental procedures had been performed.

\subsection{Administration of Quinpirole and Muscimol}

To assess the effects of administration of a dopamine D2 receptor agonist on the mechanical hypersensitivity induced by ION-CCI, an i.p. administration of $1 \mathrm{mg} / \mathrm{kg}$ quinpirole (a dopamine D2 receptor agonist, Sigma, St. Louis, MO, USA) or saline was performed 14 days after nerve ligation [27]. The head-withdrawal thresholds were measured before and 20, 60, 120, 180, and 240 min after the drug administration.

To assess the effects of the change of A11 nucleus activity on the mechanical hypersensitivity induced by ION-CCI, quinpirole or muscimol (a GABA $A_{A}$ receptor agonist, Sigma, St. Louis, MO, USA) was microinjected into the A11 nucleus 14 days after nerve ligation. At 7 days after ION-CCI, ION-CCI rats were anesthetized by i.p. administration of saline solution mixed with $2.0 \mathrm{mg} / \mathrm{kg}$ midazolam, $2.5 \mathrm{mg} / \mathrm{kg}$ butorphanol, and $0.375 \mathrm{mg} / \mathrm{kg}$ medetomidine and then placed in a stereotaxic apparatus (Narishige, Tokyo, Japan) with the incisor bar set $3.3 \mathrm{~mm}$ below the level of the ear bars. A tiny hole was made in the skull with a drill, and a metal 26-gauge stainless steel guide cannula (Bio Research Center, Aichi, Japan) was implanted for microinjection of drugs into the A11 nucleus. Stereotaxic coordinates were $3.9 \mathrm{~mm}$ caudal to the bregma, $0.5 \mathrm{~mm}$ left of the midline, and $6 \mathrm{~mm}$ ventral to the dural surface, which was $1 \mathrm{~mm}$ above the A11 injection site [15]. Screws were placed around the cannula into the skull and the cannula was affixed with dental cement. A dummy cannula was inserted into the guide cannula to ensure patency until the time of microinjection. Seven days after the implantation of the cannula, quinpirole $(20 \mu \mathrm{g}$ in $0.25 \mu \mathrm{L})$, muscimol $(0.07 \mu \mathrm{g}$ in $0.25 \mu \mathrm{L})$, or saline $(0.25 \mu \mathrm{L})$ was microinjected into the left A11 nucleus [15]. The dummy cannula was removed, and a 33-gauge internal cannula was inserted into the guide cannula. Solutions were administered through the internal cannula connected to a 1- $\mu \mathrm{L}$ Hamilton syringe via a polyethylene tube. The tip of the internal cannula was placed $3.9 \mathrm{~mm}$ caudal to the bregma, $0.5 \mathrm{~mm}$ left of the midline, and $7 \mathrm{~mm}$ ventral to the dural surface-it protruded $1 \mathrm{~mm}$ from the end of the guide cannula. The microinjections were performed slowly over a 1-min period. Head-withdrawal thresholds were measured before and 20, 40, and $60 \mathrm{~min}$ after drug administration. Measurement of head-withdrawal thresholds was performed using a blind protocol, so that the investigator measuring head-withdrawal thresholds was unaware of which experimental procedures had been performed.

\subsection{Administration of 6-OHDA and Measurement of Head-Withdrawal Threshold}

To assess the effect of destruction of dopaminergic neurons in the A11 nucleus on mechanical hypersensitivity induced by ION-CCI, 6-OHDA was microinjected into the A11 nucleus. Seven days after nerve ligation, 6-OHDA was injected into the A11 nucleus of ION-CCI rats. Thirty minutes prior to surgery, ION-CCI rats received an i.p. injection of desipramine hydrochloride $(20 \mathrm{mg} / \mathrm{kg}$, Sigma, St. Louis, MO, USA) to protect noradrenergic neurons and fibers $[48,49]$. ION-CCI rats then were anesthetized by i.p. administration of saline solution mixed with $2.0 \mathrm{mg} / \mathrm{kg}$ midazolam, $2.5 \mathrm{mg} / \mathrm{kg}$ butorphanol, and $0.375 \mathrm{mg} / \mathrm{kg}$ medetomidine and placed in a stereotaxic apparatus (Narishige, Tokyo, Japan) with the incisor bar set $3.3 \mathrm{~mm}$ below the level of the ear bars. Next, $2 \mathrm{mg}$ of 6-OHDA (Sigma, St. Louis, MO, USA) in $1 \mathrm{~mL}$ of sterile saline containing $0.01 \%$ ascorbic acid was injected into the left A11 nucleus [15,48,49]. Stereotaxic coordinates for the 6-OHDA injection site were $3.9 \mathrm{~mm}$ caudal to the bregma, $0.5 \mathrm{~mm}$ left of the midline, and $7 \mathrm{~mm}$ ventral to the dural surface. The 6-OHDA solution was administered through a 30-gauge cannula with a microinjection pump (Nihon Kohden, Tokyo, Japan) at $1 \mu \mathrm{L} / \mathrm{min}$ for $1 \mathrm{~min}$; the cannula was left in place for $5 \mathrm{~min}$ after completion of the injection. The same amount of saline was injected into ION-CCI rats for control. 
Fourteen days after the microinjection of 6-OHDA or saline into the A11 nucleus of ION-CCI rats, head-withdrawal thresholds were measured using a blind protocol, so that the investigator measuring head-withdrawal thresholds was unaware of which experimental procedures had been performed.

\subsection{Immunohistochemical Analysis}

To investigate the activity of the $V_{c}$ neurons, immunohistochemical staining for pERK was performed. Twenty minutes after the i.p. administration of quinpirole or saline as described above, ION-CCI rats received mechanical stimulation with von Frey filaments ( $15 \mathrm{~g}, 1 \mathrm{~Hz}, 5 \mathrm{~min}$ ) under deep anesthesia with pentobarbital $(80 \mathrm{mg} / \mathrm{kg})$. Mechanical stimuli were applied to the maxillary whisker pad skin on the ipsilateral side to ION-CCI. Twenty minutes after microinjection of quinpirole, muscimol, or saline into the A11 nucleus as described above, ION-CCI rats also received similar mechanical stimuli under pentobarbital anesthesia ( $n=7$ in each group), as did ION-CCI rats that were given microinjections of 6-OHDA ( $n=7$ in each group). Five minutes after mechanical stimuli, ION-CCI rats were perfused transcardially with $100 \mathrm{~mL}$ of $0.02 \mathrm{M}$ phosphate-buffered saline (PBS, $\mathrm{pH}$ 7.4) followed by $300 \mathrm{~mL}$ of $4 \%$ paraformaldehyde in a $0.1 \mathrm{M}$ phosphate buffer (PB, $\mathrm{pH} 7.4)$. Brains were removed and post-fixed overnight in the same solution and then immersed in $30 \%$ sucrose in $0.1 \mathrm{M} \mathrm{PB}$. Serial transverse sections of the brainstem were made using a freezing microtome at a thickness of $50 \mu \mathrm{m}$. Sections were incubated in $0.3 \%$ hydrogen peroxide in methanol for $20 \mathrm{~min}$, immersed in $1 \%$ normal goat serum (Vector Labs, Burlingame, CA, USA) for $30 \mathrm{~min}$, and incubated with antipERK antibody (Sigma, St. Louis, MO, USA; diluted 1:1000) for $12 \mathrm{~h}$. Sections were incubated with biotinylated rabbit antigoat antibody (Vector Labs; diluted 1:200) for $1 \mathrm{~h}$ and with avidin-biotin-peroxidase complex (Vectastain ABC Elite Kit, Vector Labs, Burlingame, CA, USA) for $1 \mathrm{~h}$; sections then were reacted with $0.05 \%$ diaminobenzidine tetrahydrochloride, $0.1 \%$ ammonium nickel sulphate, and $0.01 \%$ hydrogen peroxide in $0.05 \mathrm{M}$ Tris- $\mathrm{HCl}$ buffer ( $\mathrm{pH}$ 7.2). Sections were mounted on gelatin-coated glass slides, air-dried, and cover-slipped. As immunonegative controls, sections were processed as described above but without applying primary antibody. We examined pERK-IR cells in the superficial layers of the Vc under light microscopy. For every third section, the number of pERK-IR cells was counted in sections from the obex to a section $5 \mathrm{~mm}$ caudal to it. The five consecutive sections that contained the greatest number of pERK-IR cells in the Vc were selected in each rat ( 35 sections in each group), and the mean number of $\mathrm{pERK}-\mathrm{IR}$ cells/section was calculated.

To detect dopaminergic degeneration in the A11 nucleus following 6-OHDA microinjection, immunohistochemical staining for TH was performed in ION-CCI rats with 6-OHDA microinjection. Serial coronal sections containing the A11 cell group were made using a freezing microtome at a thickness of $50 \mu \mathrm{m}$. Sections were incubated in $0.3 \%$ hydrogen peroxide in methanol for $20 \mathrm{~min}$, immersed in $1 \%$ normal horse serum (Vector Labs, Burlingame, CA, USA) for $30 \mathrm{~min}$, and then incubated with anti-TH antibody (Sigma, St. Louis, MO, USA; diluted 1:8000) for $12 \mathrm{~h}$. Sections were then incubated with biotinylated horse antimouse antibody (Vector Labs, Burlingame, CA, USA; diluted 1:200) for $1 \mathrm{~h}$ and with avidin-biotin-peroxidase complex (Vectastain ABC Elite Kit, Vector Labs) for $1 \mathrm{~h}$; sections then were reacted with $0.05 \%$ diaminobenzidine tetrahydrochloride, $0.1 \%$ ammonium nickel sulphate, and $0.01 \%$ hydrogen peroxide in $0.05 \mathrm{M}$ Tris- $\mathrm{HCl}$ buffer (pH 7.2). Sections were mounted on gelatin-coated glass slides, air-dried, and cover-slipped. As immunonegative controls, the sections were processed with the same reagents but without applying primary antibody. For every third section, the number of TH-IR cells in the A11 nucleus were counted and the mean number of TH-IR cells/section was calculated. A blind protocol was used, so that the investigator who counted pERK-IR and TH-IR cells was unaware of the experimental procedures performed.

\subsection{Statistical Analysis}

Behavioral data are shown as box and whisker plots. The bottom and top of the box indicate the first and third quartiles, respectively. The end of the whiskers represents the minimum and maximum values of all data. If no median is shown in the box, the median is at the top or bottom of the box. 
If whisker is not displayed in the box, the maximum and minimum values match the first and third quartiles. Other data are shown as means \pm SEM. SPSS statistics (ver. 24, IBM, Chicago, IL, USA) was used for statistical analyses. Differences of head-withdrawal thresholds were analyzed using a two-way repeated measures analysis of variance (ANOVA) followed by Bonferroni corrections for multiple comparisons as post hoc comparison. For the evaluation of immunohistochemical data, we performed a two-way ANOVA followed by Bonferroni tests for multiple comparisons (TH-IR cells), a one-way ANOVA followed by Bonferroni tests for multiple comparisons (pERK-IR cells in the rats with microinjection of quinpirole or muscimol into the A11 nucleus), and a t-test (pERK-IR cells in rats with i.p. drug administration and microinjection of 6-OHDA into the A11 nucleus). $p<0.05$ was considered statistically significant.

Supplementary Materials: The following are available online at http://www.mdpi.com/1422-0067/21/6/1945/s1, File S1: Raw data of behavioral and immunohistochemical responses., Figure S1: Photomicrograph of pERK-IR cells in the $V_{c}$ in an ION-CCI rat that received i.p. administration of saline., Figure S2: Photomicrograph of pERK-IR cells in the Vc in an ION-CCI rat that received i.p. administration of quinpirole., Figure S3: Photomicrograph of a microinjection site within the A11 nucleus (low magnification)., Figure S4: Photomicrograph of a microinjection site within the A11 nucleus (high magnification)., Figure S5: Photomicrograph of pERK-IR cells in the Vc in an ION-CCI rat that received microinjection of saline., Figure S6: Photomicrograph of pERK-IR cells in the Vc in an ION-CCI rat that received microinjection of quinpirole., Figure S7: Photomicrograph of pERK-IR cells in the Vc in an ION-CCI rat that received microinjection of muscimol., Figure S8: Photomicrograph of TH-IR cells in the A11 nucleus in an ION-CCI rat that received microinjection of 6-OHDA (low magnification)., Figure S9: Photomicrograph of TH-IR cells in the A11 nucleus in an ION-CCI rat that received microinjection of 6-OHDA (high magnification)., Figure S10: Photomicrograph of pERK-IR cells in the Vc in an ION-CCI rat that received microinjection of saline into the A11 nucleus., and Figure S11: Photomicrograph of pERK-IR cells in the Vc in an ION-CCI rat that received microinjection of 6-OHDA into the A11 nucleus.

Author Contributions: Conceptualization, H.M. and H.N.; methodology, H.M. and H.N.; software, H.M.; validation, H.M., N.U.; H.H.; C.K. and H.N.; formal analysis, H.M.; investigation, H.M. and N.U.; resources, H.M.; data curation, H.M. and N.U.; writing-original draft preparation, H.M.; writing-review and editing, H.M.; N.U.; H.H. and C.K.; visualization, H.M.; supervision, H.M. and H.N; project administration, H.M. and H.N.; funding acquisition, H.M. All authors have read and agreed to the published version of the manuscript.

Funding: This research was funded by JSPS KAKENHI, grant number 17K17242, 19K19192.

Acknowledgments: The authors thank Enago (www.enago.jp) for English language editing.

Conflicts of Interest: The authors declare no conflict of interest. The funders had no role in the design of the study; in the collection, analyses, or interpretation of data; in the writing of the manuscript, or in the decision to publish the results.

\section{References}

1. Meyer, D.J.; Wolfle, T.L.; Akil, H.; Carder, B.; Liebeskind, J.C. Analgesia from electrical stimulation in the brainstem of the rat. Science 1971, 174, 1351-1354. [CrossRef] [PubMed]

2. Fields, H.L.; Heinricher, M.M. Anatomy and physiology of a nociceptive modulatory system. Philos. Trans. R. Soc. Lond. B Biol. Sci. 1985, 308, 361-374. [PubMed]

3. Fields, H.L.; Basbaum, A.I.; Heinricher, M.M. Central nervous system mechanisms of pain modulation. In Wall and Melzack's Textbook of Pain, 5th ed.; McMahon, S., Koltzenberg, M., Eds.; Elsevier: London, UK, 2006; pp. 125-142.

4. Heinricher, M.M.; Tavares, I.; Leith, J.L.; Lumb, B.M. Descending control of nociception: Specificity, recruitment and plasticity. Brain Res. Rev. 2009, 60, 214-225. [CrossRef] [PubMed]

5. Chudler, E.H.; Dong, W.K. The role of the basal ganglia in nociception and pain. Pain 1995, 60, 3-38. [CrossRef]

6. Millan, M.J. Descending control of pain. Prog. Neurobiol. 2002, 66, 355-474. [CrossRef]

7. Paalzow, G.H. L-DOPA induces opposing effects on pain in intact rats: (-)-sulpiride, SCH2339 or alpha-methyl-DL-p-tyrosine methylester hydrochloride reveals profound hyperalgesia in large antinociceptive doses. J. Pharmacol. Exp. Ther. 1992, 263, 470-479.

8. Hagelberg, N.; Jaaskelainen, S.K.; Martikainen, I.K.; Mansikka, H.; Forssell, H.; Scheinen, H.; Hietala, J.; Pertovaara, A. Striatal dopamine D2 receptors in modulation of pain in humans: A review. Eur. J. Pharmacol. 2004, 500, 187-192. [CrossRef] 
9. Shimizu, T.; Iwata, S.; Morioka, H.; Masuyama, T.; Fukuda, T.; Nomoto, M. Antinociceptive mechanism of L-DOPA. Pain 2004, 110, 246-249. [CrossRef]

10. Fleetwood-Walker, S.M.; Hope, P.J.; Mitchell, R. Antinociceptive actions of descending dopaminergic tracts on cat and rat dorsal horn somatosensory neurons. J. Physiol. (London) 1988, 399, 335-348. [CrossRef]

11. Yang, H.W.; Zhou, L.J.; Hu, N.W.; Xin, W.J.; Liu, X.G. Activation of spinal D1/D5 receptors induces late-phase LTP of C-fiber-evoked field potentials in rat spinal dorsal horn. J. Neurophysiol. 2005, 94, 961-967. [CrossRef]

12. Chen, J.F.; Qin, Z.H.; Szele, F.; Bai, G.; Weiss, B. Neuronal localization and modulation of the D2 dopamine receptor mRNA in brain of normal mice and mice lesioned with 6-hydroxydopamine. Neuropharmacology 1991, 30, 927-941. [CrossRef]

13. Bregerot, A.; Storer, R.J.; Goadsby, P.J. Dopamine inhibits trigeminovascular transmission in the rat. Ann. Neurol. 2007, 61, 251-262. [CrossRef] [PubMed]

14. Zhu, H.; Clemens, S.; Sawchuk, M.; Hochman, S. Expression and distribution of all dopamine receptor subtype $\left(\mathrm{D}_{1}-\mathrm{D}_{5}\right)$ in the mouse lumber spinal cord: A real-time polymerase chain reaction and non-autoradiographic in situ hybridization study. Neuroscience 2007, 149, 885-897. [CrossRef] [PubMed]

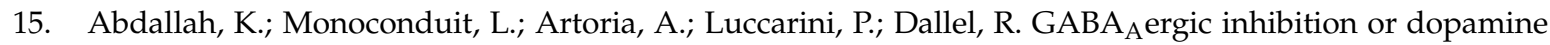
denervation of the A11 hypothalamic nucleus induces trigeminal analgesia. Pain 2015, 156, 644-655. [CrossRef]

16. Charbit, A.R.; Akerman, S.; Holland, P.R.; Goadsby, P.J. Neurons of the dopaminergic/calcitonin gene-related peptide A11 cell group modulate neuronal firing in the trigeminocervical complex: An electrophysiological and immunohistochemical study. J. Neurosci. 2009, 29, 12532-12541. [CrossRef]

17. Wei, H.; Viisanen, H.; Petrovaara, A. Descending modulation of neuropathic hypersensitivity by dopamine D2 receptors in or adjacent to the hypothalamic A11 cell group. Pharmacol. Res. 2009, 59, 355-363. [CrossRef]

18. Liu, S.; Tang, Y.Y.; Shu, H.; Tatum, D.; Bai, Q.; Crawford, J.; Xing, Y.; Lobo, M.K.; Bellinger, L.; Kramer, P.; et al. Dopaminergic receptor D2, but not D1, mediates descending dopaminergic pathway-produced analgesic effect in a trigeminal neuropathic pain mouse model. Pain 2019, 160, 334-344. [CrossRef]

19. Ji, R.R.; Baba, H.; Brenner, G.J.; Woolf, C.J. Nociceptive-specific activation of ERK in spinal neurons contributes to pain hypersensitivity. Nat. Neurosci. 1999, 2, 1114-1119. [CrossRef]

20. Shibuta, K.; Suzuki, I.; Shinoda, M.; Tsuboi, Y.; Honda, K.; Sessle, B.J.; Iwata, K. Organization of hyperactive microgrial cells in trigeminal spinal subnucleus caudalis and upper cervical spinal cord associated with orofacial neuropathic pain. Brain Res. 2012, 1451, 74-86. [CrossRef]

21. Paalzow, G.H.; Paalzow, L.K. Opposing effects of apomorphine in pain in rats. Evaluation of the dose-response curve. Eur. J. Pharmacol. 1983, 88, 27-35. [CrossRef]

22. Barasi, S.; Duggal, K.N. The effect of local and systemic application of dopaminergic agents on tail flick latency in the rat. Eur. J. Pharmacol. 1985, 117, 287-294. [CrossRef]

23. Zarrindast, M.R.; Nassiri-Rad, S.; Pazouki, M. Effects of dopaminergic agents on antinociception in formalin test. Gen. Pharmacol. 1999, 32, 517-522. [CrossRef]

24. Pelissier, T.; Laurido, C.; Hernandez, A.; Condtandil, L.; Eschalier, A. Biphasic effect of apomorphine on rat nociception and effect of dopamine D2 receptor antagonists. Eur. J. Pharmacol. 2006, 546, 40-47. [CrossRef] [PubMed]

25. Munro, G. Dopamine D1and D2 receptor agonism enhances antinociception mediated by the serotonin and noradrenaline reuptake inhibitor duloxetine in the rat formalin test. Eur. J. Pharmacol. 2007, 575, 66-74. [CrossRef] [PubMed]

26. Gao, X.; Zhang, Y.; Wu, G. Effects of dopaminergic agents on carrageenan hyperalgesia in rats. Eur. J. Pharmacol. 2000, 406, 53-58. [CrossRef]

27. Cobacho, N.; de la Calle, J.L.; Paino, C.L. Dopaminergic modulation of neuropathic pain: Analgesia in rats by a D2-type receptor agonist. Brain Res. Bull. 2014, 106, 62-71. [CrossRef]

28. Bouthenet, M.L.; Martres, M.P.; Sales, N.; Schwartz, J.C. A detailed mapping of dopamine D-2 receptors in rat central nervous system by autoradiography with $\left[{ }^{125} \mathrm{I}\right]$ iodosulpride. Neuroscience 1987, 20, 117-155. [CrossRef]

29. Lapirot, O.; Melin, C.; Modolo, A.; Nicolas, C.; Messaoudi, Y.; Monconduit, L.; Artola, A.; Luccarini, P.; Dallel, R. Tonic and phasic descending dopaminergic controls of nociceptive transmission in the medullary dorsal horn. Pain 2011, 152, 1821-1831. [CrossRef]

30. Ansah, O.; Leite-Almeida, H.; Wei, H.; Petrovaara, A. Striatal dopamine D2 receptors attenuate neuropathic hypersensitivity in the rat. Exp. Neurol. 2007, 205, 536-546. [CrossRef] 
31. Skageberg, G.; Lindvall, O. Organization of diencephalic dopamine neurons projecting to the spinal cord in the rat. Brain Res. 1985, 342, 340-351. [CrossRef]

32. Pappas, S.S.; Tiernan, C.T.; Behrouz, B.; Jordan, C.L.; Breedlove, S.M.; Goudreau, J.L.; Lookingland, K.J. Neonatal androgen-dependent sex differences in lumber spinal cord dopamine concentrations and the number of A11 diencephalospinal dopamine neurons. J. Comp. Neurol. 2010, 518, 2423-2436. [PubMed]

33. Baud, P.; Mayo, W.; Le Moral, M.; Simon, H. Locomotor hypersensitivity in the rat after infusion of muscimol and [D-Ala] Metenkephalin into the nucleus basalis magnocellularis. Possible interaction with cortical cholinergic protection. Brain Res. 1988, 452, 203-211. [CrossRef]

34. Van den Bos, R.; Cools, A.R. The ventral pallidum/substantia innominata complex: Further evidence for heterogeneity as determined by the effects of GABA-ergic drugs. Behav. Pharmacol. 1989, 1, 29.

35. Johnson, E.F.; Szechtman, H. A dose-response study of separate and combined effects of the serotonin agonist 8-OT-DPAT and the dopamine agonist quinpirole on locomotor sensitization, cross-sensitization, and conditioned activity. Bahav. Pharmacol. 2016, 27, 439-450. [CrossRef] [PubMed]

36. Petrile, R.A.N.; Corvino, M.E.; Marchette, R.C.N.; Pavesi, E.; Cavalli, J.; Ramos, A.; Izidio, G.S. The quinpirole hypolocomotive effects are strain and route of administration dependent in SHR and SLA16 isogenic rats. Behav. Genet. 2017, 47, 552-563.

37. Paulus, W.; Trenkwalder, C. Less is more: Pathophysiology of dopaminergic-therapy-related augmentation in restless legs syndrome. Lancet Neurol. 2006, 5, 878-886. [CrossRef]

38. Clemens, S.; Hochman, S. Conversion of the modulatory actions of dopamine on spinal reflexes from depression to facilitation in D3 receptor knock-out mice. J. Neurosci. 2004, 24, 11337-11345. [CrossRef]

39. Clemens, S.; Belin-Rauscent, A.; Simmers, J.; Combes, D. Opposing modulatory effects of D1- and D2-like receptor activation on a spinal central pattern generator. J. Neurophysiol. 2012, 107, 2250-2259. [CrossRef]

40. Aira, Z.; Barrenetxea, T.; Busea, I.; Gomez-Esteban, J.C.; Azuke, J.J. Synaptic upregulation and superadditive interaction of dopamine D2- and $\mu$-opioid receptors after peripheral nerve injury. Pain 2014, 155, 2526-2533. [CrossRef]

41. Xu, W.; Zhang, J.; Wang, Y.; Wang, L.; Wang, X. Changes in the expression of voltage-gated sodium channels $\mathrm{Na}_{V} 1.8$, and $\mathrm{Na}_{V} 1.9$ in the rat trigeminal ganglia following chronic constriction injury. Neuroreport 2016, 27, 929-934. [CrossRef]

42. Imamura, Y.; Kawamoto, H.; Nakanishi, O. Characterization of heat-hyperalgesia in an experimental trigeminal neuropathy in rats. Exp. Brain Res. 1997, 116, 97-103. [CrossRef]

43. Mercado-Reyes, J.; Almanza, A.; Segura-Chama, P.; Pellicer, F.; Mercado, F. D2-like receptor agonist synergizes the $\mu$-opioid agonist spinal antinociception in nociceptive, inflammatory and neuropathic models of pain in the rat. Eur. J. Pharmacol. 2019, 853, 56-64. [CrossRef] [PubMed]

44. Aira, Z.; Barrenetxea, T.; Bueas, I.; Garcia del Cano, G.; Azkue, J.J. Dopamine D1-like receptors regulate constitutive, $\mu$-opioid receptor-mediated repression of use-dependent synaptic plasticity in dorsal horn neurons: More harm than good? J. Neurosci. 2016, 36, 5661-5673. [CrossRef] [PubMed]

45. Dai, W.I.; Xiong, F.; Yan, B.; Cao, Z.Y.; Liu, W.T.; Liu, J.H.; Yu, B.Y. Blockade of neuronal dopamine D2 receptor attenuates morphine tolerance in mice spinal cord. Sci. Rep. 2016, 6, 38746. [CrossRef] [PubMed]

46. Zimmermann, M. Ethical guidelines for investigations of experimental pain in conscious animals. Pain 1983, 16, 109-110. [CrossRef]

47. Suzuki, I.; Tsuboi, Y.; Shinoda, M.; Shibuta, K.; Honda, K.; Katagiri, A.; Kiyomoto, M.; Sessle, B.J.; Matsuura, S.; Ohara, K.; et al. Involvement of ERK phosphorylation of trigeminal spinal subnucleus caudalis neurons in thermal hypersensitivity in rats with infraorbital nerve injury. PLoS ONE 2013, 8, e57278. [CrossRef] [PubMed]

48. Maegawa, H.; Morimoto, Y.; Kudo, C.; Hanamoto, H.; Boku, A.; Sugimura, M.; Kato, T.; Yoshida, A.; Niwa, H. Neural mechanism underlying hyperalgesic response to orofacial pain in Parkinson's disease model rats. Neurosci. Res. 2015, 96, 59-68. [CrossRef] [PubMed]

49. Maegawa, H.; Adachi, N.; Hanamoto, H.; Kudo, C.; Niwa, H. Bilateral Parkinson's disease model rats exhibit hyperalgesia to subcutaneous formalin administration into the vibrissa pad. PLOS ONE 2019, 14, e0225928. [CrossRef]

(C) 2020 by the authors. Licensee MDPI, Basel, Switzerland. This article is an open access article distributed under the terms and conditions of the Creative Commons Attribution (CC BY) license (http://creativecommons.org/licenses/by/4.0/). 